\title{
Acute Cholecystitis due to a gall bladder torsion: A case report
}

\author{
Dhafer Hadded ${ }^{1}$, MERYAM MESBAHI ${ }^{1}$, Mona Cherif ${ }^{1}$, Yazid Benzarti ${ }^{1}$, Yecine Jemli $^{1}$, \\ Chiraz Chammakhi ${ }^{1}$, and Anis Ben Maamer ${ }^{1}$ \\ ${ }^{1}$ Habib Thameur Hospital
}

May 14, 2021

\begin{abstract}
Gallbladder torsion is an unusual cause of an acute abdomen that can be mortal. It mimicks commonly acute cholecystitis. Its diagnosis is challenging for surgeons. Once it is suspected, prompt surgical treatment must be carried out, which is detorsion with cholecystectomy.
\end{abstract}

Acute Cholecystitis due to a gall bladder torsion: A case report ABSTRACT:

Gallbladder torsion is an unusual cause of an acute abdomen that can be mortal. It mimicks commonly acute cholecystitis. Its diagnosis is challenging for surgeons. Once it is suspected, prompt surgical treatment must be carried out, which is detorsion with cholecystectomy.

Keywords: cholecystitis, gallbladder torsion, cholecystectomy, gall bladder volvulus,acute abdomen, laparoscopic cholecystectomy

INTRODUCTION:

Gallbladder torsion is a rare phenomenon of an acute abdomen. It was first described in 1898 by Wendel in a 25-year-old pregnant woman[1]. It happens when adhesion between the gallbladder and liver is lost. The cause of torsion is usually due to underlying anatomical variations. Acute cholecystitis is the most common presentation of gall bladder volvulus. the preoperative diagnosis presents a dilemma[2]. We report a rare case of gallbladder volvulus in a young age male diagnosed preoperatively as acute cholecystitis. Widely patients are falsely diagnosed with acute cholecystitis and there is a delay in surgical treatment so we hope to promote awareness and encourage prompt surgical management in patients with suspected gallbladder torsion.

\section{CASE REPORT:}

A26 year-old man with no medical or surgical or drug history was admitted to our Surgical department for cramped abdominal pain in the right hypochondrium associated with vomiting. This pain had been present for 24 hours and was of increasing intensity. There was no fever neither jaundice. Vital parameters were normal. The abdominal exam showed tenderness in the right upper quadrant. Laboratory tests showed white blood cells at 14470/mm3, a C-reactive protein at $3 \mathrm{mg} / \mathrm{L}$, with no alteration of the liver; and pancreatic tests.

Ultrasound was initially performed, demonstrating double-wall thickening of the gallbladder with no distention associated nor gallstones (figure 1). CT scan was performed confirming gallbladder diffuse wall thickening, an abrupt tapering of the cystic duct and cystic artery (figure 2). A preoperative diagnosis of 
gallbladder torsion was suspected, so the decision was taken to perform an exploratory laparoscopy. Intraoperatively the gall bladder was gangrenous, was hanging freely and a torsion around the gallbladder axis less than $180^{\circ}$ was noted in the peritoneum cavity and was attached only along the gall bladder neck (figure 3), detorsion was done manually by flange section, and cholecystectomy was carried out (figure4). the post-operative recovery occurred without incidents.

\section{DISCUSSION:}

Acute cholecystitis is a common emergent pathology, which is rarely due to Gallbladder torsion. This mechanism can be explaining by ischemia of its wall than necrosis [3].

Its etiology is not yet been identified, factors studied in the literature involved anatomical abnormalities such as a long gallbladder mesentery, providing it the possibility to tilt freely from the liver bed and easily twist upon itself. [4]

The mechanical events may be suddenly changing in body position, violent peristalsis of adjacent internal organs, and blunt injuries. Studies have shown that level elevation of cholecystokinin causing by gallbladder peristalsis after a fatty meal may conclude to gallbladder torsion[5].

This is due to a gallbladder without adhesion to the liver, concluding to the torsional movement. Referring to the literature: The Gross classification studied the types of gallbladder mobility according to the adherence with the life concluding to two types. Type I corresponds to the attachment of the gallbladder and cystic duct to the lower surface of the liver through the mesentery. In Type II, the cystic duct alone is attached to the liver[3]

Clinical symptoms are not distinctive and could comprise abdominal pain, nausea, and vomiting, and a palpable mass. A triple triad has been identified to clinically dissimilate between acute cholecystitis and gallbladder volvulus: the first is patient features, The second is based on clinical signs of intense onset pain, right upper quadrant pain, and vomiting, The third is of clinical characteristics on physical examination: a palpable mass in the right upper quadrant[1].

laboratory data of gallbladder torsion often indicate elevated inflammatory response. However there is a normal liver function, such in our case.

Ultrasound is viewed as the first mean of diagnosis and often revealing a large floating gallbladder without gallstones, and a thickened gallbladder wall[6]. Other more specific signs can be seen such as the presence of the gallbladder outside its normal anatomic fossa and a knot sign [6].

CT scan is viewed as the first means for the diagnosis and it usually described a swollen gallbladder with wall thickening and no gallstones[2], often associated with the abrupt tapering of the cystic duct [2],indirect signs of the necrosing process which are pericholecystic fluid and thumb printing of the gallbladder [6]. Also, it can demonstrate a free-floating gallbladder such as Whirl Sign and cystic duct knot sign, which are surely pathognomonic for gallbladder torsion[6].

Treatment of gallbladder volvulus is emergency cholecystectomy, as its delay might increase the risk of perforation, peritonitis, and death.

the laparoscopic approach is the gold standard and it should begin with a release of the torsion, thus avoiding damage to the main bile duct[3].

Delaying diagnosis and treatment can result in bilious peritonitis, which would increase mortality.

\section{Conclusion:}

Diagnosis of gallbladder volvulus is certainly challenging. Once it is suspected, speedy surgical treatment must be carried out. This approach may prevent the higher mortality and morbidity associated with gallbladder torsion due to perforation, and biliary peritonitis.

Conflict of interest for all authors: The authors declare no competing interest. 
Funding : None.

Compliance with Ethical Standards: The patient has provided both verbal and written consent for the publication of This article. It was made sure that his identity will be kept a secret at all levels.

Consent: Written informed consent was taken from the patient regarding the publication of this case report. It was made sure that his identity will be kept a secret at all levels. A copy of a written request is available for review if requested.

Acknowledgments: There were no acknowledgments to mention it.

Author contributions : All authors were involved in the researching, writing, and editing of the manuscript.

\section{References:}

[1] C. Abou Sleiman et al. , « Gallbladder Volvulus: An Unusual Presentation », Am. J. Case Rep., vol. 20, p. 1879-1882, dec. 2019, doi: 10.12659/AJCR.916233.

[2] T. Bekki et al. , ii Complete torsion of gallbladder following laparoscopic cholecystectomy: A case study i¿, Int. J. Surg. Case Rep. , vol. 37, p. 257-260, 2017, doi: 10.1016/j.ijscr.2017.06.051.

[3] M. David, B. Mattia, G. Seda, N. Benjamin, et H. Jerome, ii Gallbladder torsion: a cholecystectomy that cannot be delayed ¿i, Acta Chir. Belg. , vol. 120, $\mathrm{n}^{\mathrm{o}} 4$, p. 279-281, juill. 2020, doi: 10.1080/00015458.2018.1553819.

[4] Elizabeth E Price, ii OUP accepted manuscript i., J. Surg. Case Rep. , 2019, doi: 10.1093/jscr/rjz221.

[5] W. Farhat et al. , ii Gallbladder volvulus: A case report and review of the literature i¿, Int. J. Surg. Case Rep. , vol. 60, p. 75-78, 2019, doi: 10.1016/j.ijscr.2019.02.025.

[6] N. Matsuhashi, ii Volvulus of the gall bladder diagnosed by ultrasonography, computed tomography, coronal magnetic resonance imaging and magnetic resonance cholangio-pancreatography i $i$, World J. Gastroenterol. , vol. 12 , $\mathrm{n}^{\circ}$ 28, p. 4599, 2006, doi: 10.3748/wjg.v12.i28.4599.

Figure 1: Ultrasound showing double-wall thickening of the gallbladder with trace pericholecystic fluid with no distention

Figure 2: Abdominal CT revealed gallbladder wall thickening with an abrupt tapering of the cystic duct and cystic artery

Figure 3: Intraoperative imaging showing a large distended gangrenous cholecystitis complicating gallbladder volvulus

Figure4: Intraoperative imaging showing: detorsion was done manually by the flange section, and Cystic duct and cystic artery were identified by dissection, they were ligated and cut.
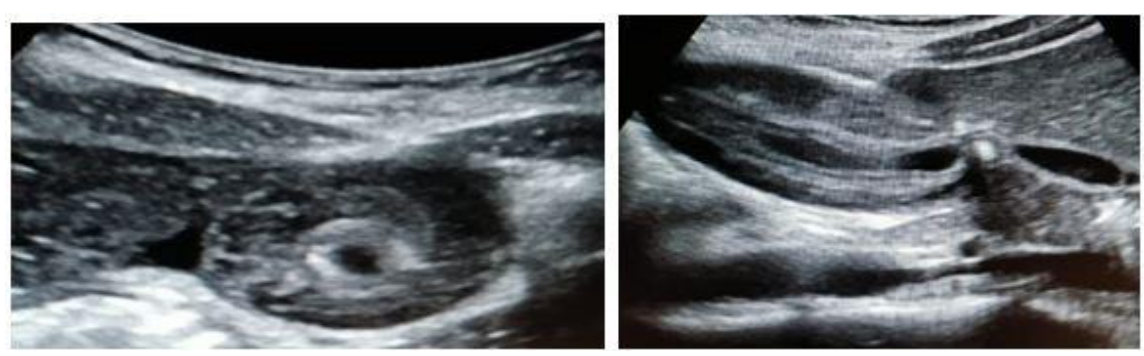

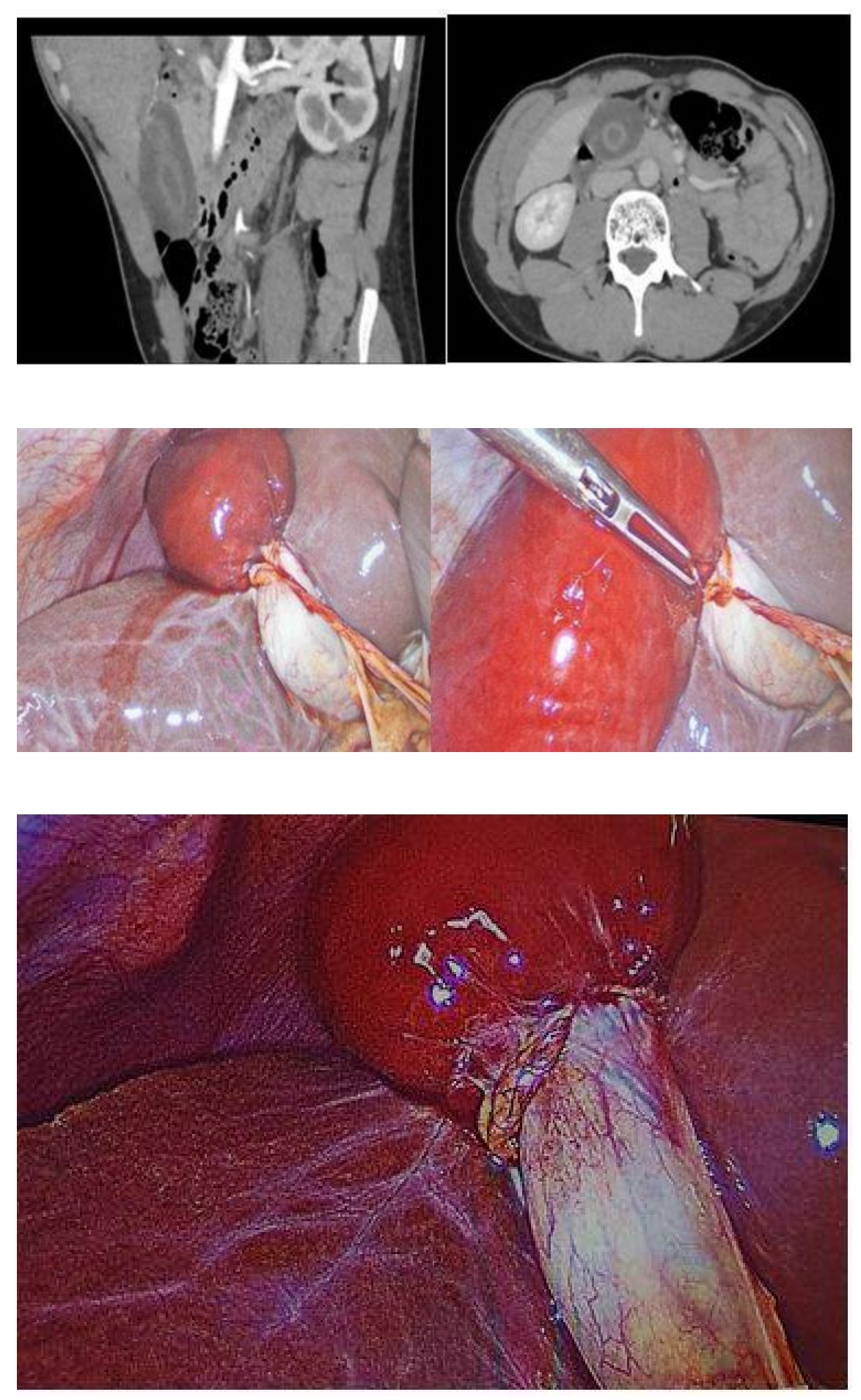